\title{
S-Implications on Complete Lattices and the Interval Constructor
}

R.H.S. REISER ${ }^{1}$, G.P. DIMURO ${ }^{2}$, Programa de Pós-Graduação em Informática, UCPel, 96010-000 Pelotas, Brazil

B.C. BEDREGAL ${ }^{3}$, H.S. SANTOS ${ }^{4}$, Departamento de Informática e Matemática Aplicada, UFRN, 59072-970 Natal, Brazil

R. CALlEJAS-BEDREGAL ${ }^{5}$ Departamento de Matemática, UFPB, 58051900 João Pessoa, Brazil.

\begin{abstract}
The aim of this work is to present an approach of interval fuzzy logic based on complete lattices. In particular, we study the extensions of the notions of t-conorms, fuzzy negations and S-implication, from the unit interval to arbitrary complete lattices. Some general properties of S-implications on complete lattices are analyzed. We show that the interval extensions of t-conorms, fuzzy negations and S-implications on complete lattices preserve the optimality property, being the best interval representations of these fuzzy connectives.
\end{abstract}

Keywords. S-implications, Interval Constructor, Complete Lattices.

\section{Introduction}

Fuzzy set theory is a mathematical theory introduced by Zadeh [32] to deal with uncertain or vague notions, using values in the unit interval $[0,1]$ to indicate the specialist's uncertainty when evaluating the membership degree of an element to a given set. Fuzzy Logic, the logic underlying this theory, allows more human-like interpretation and reasoning in machines than the classic logic. Fuzzy systems technology has achieved its maturity with widespread applications in many areas, such as, e.g., control, automation, artificial intelligence, image/signal processing, pattern recognition. [23]

On the other hand, interval mathematics [25] was developed to model the uncertainty in numerical computations, allowing the development of reliable computational tools with the rigorous and automatic error analysis of numerical algorithms, with applications in technological and scientific computations [3, 11, 19].

\footnotetext{
${ }^{1}$ reiser@ucpel.tche.br

${ }^{2}$ liz@ucpel.tche.br

${ }^{3}$ bedregal@dimap.ufrn.br

${ }^{4}$ lda@digizap.com.br

${ }^{5}$ roberto@mat.ufpb.br
} 
There are several papers connecting these areas (see, e.g., $[9,12,14,22,24]$ ), showing the intrinsic relation between fuzzy logic and interval mathematics. In this paper, we consider the so-called interval fuzzy logic approach, which uses interval as membership degrees of fuzzy sets, aiming at dealing with the uncertainty associated to digital computers.

Lattice theory has been used to consider fuzzy logic in a more general framework. See, e.g., the works on $L$-fuzzy set theory [15], BL-algebras of Hájek [16] and Brouwerian lattices [29]. The notion of triangular norm (t-norm, for short) of partially ordered sets, which are more general mathematical structures than complete lattices, is considered in $[8,2]$. In $[26,4]$, an extension of t-norms for bounded lattice was presented in the same sense as proposed by $[8,2]$. In $[9,10]$ complete lattices were considered.

This work uses complete lattices in the interval fuzzy logic approach. Different from the papers mentioned in the above paragraph, the aim of this work is to introduce a generalization of t-conorms, fuzzy negations and fuzzy S-implications for arbitrary complete lattices. It is shown that the interval constructor preserves the usual constructions of fuzzy S-implication as well as the related properties. We observe that these results are based on the preservation of the monotonicity of such functions by their interval representations.

The paper is organized as follows. In Sect. 2., we review the basic concepts, such as complete lattices and the interval version of this class of lattices. In Sect. 3., we present the interval constructor and discuss the conditions to obtain the best interval representation of a real function. Interval extensions of fuzzy t-conorms and fuzzy negations are introduced in Sections 4. and 5., respectively. Further analysis of the properties satisfied by fuzzy S-implications is done in Sect. 6.: Sect. 6.1. shows that the minimal properties of fuzzy implications may be extended considering interval fuzzy degrees, in a natural way; in Sect. 6.2., a commutative diagram relating fuzzy

S-implications with interval fuzzy S-implications is discussed. Section 7. is the Conclusion.

\section{Lattices}

Let $\mathbf{L}=\langle L, \wedge, \vee\rangle$ be an algebraic structure, where $L$ is a nonempty set and $\wedge$ and $\vee$ are binary operations, called, respectively, meet and join. $\mathbf{L}$ is a lattice, if, for each $x, y, z \in L$, the operations meet and join are commutative, associative, and satisfy the absorption laws:

$$
\begin{array}{ll}
\text { (1a) } x \wedge y=y \wedge x ; & \text { (1b) } x \vee y=y \vee x ; \\
\text { (2a) } x \wedge(y \wedge z)=(x \wedge y) \wedge z ; & \text { (2b) } x \vee(y \vee z)=(x \vee y) \vee z ; \\
\text { (3a) } x \wedge(x \vee y)=x ; & \text { (3b) } x \vee(x \wedge y)=x
\end{array}
$$

If there exist two distinct elements, 0 and 1 , such that, for each $x \in L, x \wedge 1=x$ and $x \vee 0=x$, then $\mathbf{L}=\langle L, \wedge, \vee, 1,0\rangle$ is said to be a bounded lattice.

Each lattice $\mathbf{L}=\langle L, \wedge, \vee\rangle$ establishes a partial order $\leq_{L} \subseteq L \times L$, defined by

$$
x \leq_{L} y \Leftrightarrow x \wedge y=x \Leftrightarrow x \vee y=y,
$$


where $\wedge$ is the greatest lower bound (infimum) and $\vee$ is the least upper bound (supremum) of the set $\{x, y\}$. A bounded lattice $\mathbf{L}$ is called complete if the infimum and the supremum exist for any subset of $L$.

In the interval version of a complete lattice $\mathbf{L}$, denoted by $\mathbb{I} \mathbf{L}=\langle\mathbb{L}, \wedge, \vee,[1,1],[0,0]\rangle$, the set of intervals in $L, \mathbb{L}=\left\{[\underline{x}, \bar{x}]: \underline{x}, \bar{x} \in L, \underline{x} \leq_{L} \bar{x}\right\}$, satisfies the conditions

(a) $[\underline{x}, \bar{x}] \wedge[\underline{y}, \bar{y}]=\left[\underline{x} \wedge_{L} \underline{y}, \bar{x} \wedge_{L} \bar{y}\right] \quad$ and $\quad$ (b) $[\underline{x}, \bar{x}] \vee[\underline{y}, \bar{y}]=\left[\underline{x} \vee_{L} \underline{y}, \bar{x} \vee_{L} \bar{y}\right]$.

Clearly, $\mathbb{I} \mathbf{L}$ is also a complete lattice. In fact, considering $A \subset \mathbb{L}$, it holds that

$$
\begin{aligned}
\sup A & =[\sup \{\underline{X} \mid[\underline{X}, \bar{X}] \in A\}, \sup \{\bar{X} \mid[\underline{X}, \bar{X}] \in A\}], \\
\inf A & =[\inf \{\underline{X} \mid[\underline{X}, \bar{X}] \in A\}, \inf \{\bar{X} \mid[\underline{X}, \bar{X}] \in A\}] .
\end{aligned}
$$

The associated order for $\mathbb{I} \mathbf{L}$ agrees with the product order and can be expressed as

$$
[\underline{x}, \bar{x}] \leq_{\mathbb{L}}[\underline{y}, \bar{y}] \text { if and only if } \underline{x} \leq_{L} \underline{y} \text { and } \bar{x} \leq_{L} \bar{y} .
$$

The partial order defined in Eq. (2.1) generalizes the most usual partial order in the context of interval mathematics, introduced by Kulisch and Miranker [21]. That is, when the usual order of the real numbers is considered and $\mathbf{L}$ is the unitary interval, the order in Eq. (2.1) coincides with the order of Kulisch-Miranker.

Remark 1. One can notice that:

(i) $X \leq_{\mathbb{L}} Y$ if and only if, for each $x \in X$, there exists $y \in Y$ such that $x \leq_{L} y$ and, for each $y \in Y$, there exists $x \in X$ such that $x \leq_{L} y$.

(ii) $X=Y$ if and only if, for each $x \in X$, there exists $y \in Y$ such that $x \leq_{L} y$ and, for each $y \in Y$, there exists $x \in X$ such that $y \leq_{L} x$.

\section{Interval Representations}

The notions of interval representation and canonical interval representation, here refereed as the best interval representation, were both introduced in [28], with the goal of providing a mathematical foundation for interval computations, where correctness and optimality principles can be verified. In this section, a generalization for arbitrary complete lattices is presented. Notice that an interval $[\underline{x}, \bar{x}] \in \mathbb{L}$ may be seen either as a pair of elements $(\underline{x}, \bar{x})$ or as a set of elements $\left\{\alpha \in L \mid \underline{x} \leq_{L} \alpha \leq_{L} \bar{x}\right\}$, and the latter is considered in this paper.

An interval $X \in \mathbb{L}$ is said to be an interval representation of each $\alpha \in X$. Let $X$ and $Y$ be interval representations of $\alpha$. Thus, $X$ is said to be a better representation of $\alpha$ than $Y$ if $X \subseteq Y$. This notion can be easily extended for $n$-tuples of intervals, indicated by $(\vec{X})=\left(X_{1}, \ldots, X_{n}\right)$.

Definition 3.1. Let $L$ be a complete lattice. A function $F: \mathbb{L}^{n} \longrightarrow \mathbb{L}$ is an interval representation of a function $f: L^{n} \longrightarrow L$ if, for each $\vec{X} \in \mathbb{L}^{n}$ and $\vec{x} \in \vec{X}$, $f(\vec{x}) \in F(\vec{X})$.

Thus, when $L$ is a complete lattice, an interval function $F: \mathbb{L}^{n} \longrightarrow \mathbb{L}$ is a better interval representation of the function $f: L^{n} \longrightarrow L$ than $G: \mathbb{L}^{n} \longrightarrow \mathbb{L}$, denoted by $G \sqsubseteq F$, if for each $\vec{X} \in \mathbb{L}^{n}$, the inclusion $F(\vec{X}) \subseteq G(\vec{X})$ holds. 
Definition 3.2. Let $L$ be a complete lattice. For each function $f: L^{n} \longrightarrow L$, the interval function $\widehat{f}: \mathbb{L}^{n} \longrightarrow \mathbb{L}$, defined by

$$
\widehat{f}(\vec{X})=[\inf \{f(\vec{x}) \mid \vec{x} \in \vec{X}\}, \sup \{f(\vec{x}) \mid \vec{x} \in \vec{X}\}]
$$

is called the best interval representation of $f$.

The interval function $\widehat{f}$ is well defined $[27,28]$ and for any other interval representation $F$ of $f, F \sqsubseteq \widehat{f}$. The function $\widehat{f}$ returns a narrower interval than any other interval representation of $f$ [17]. Thus, $\widehat{f}$ has the optimality property of interval algorithms mentioned by Hickey et al. [18], when it is seen as an algorithm to compute a real function $f$.

\section{T-conorms on Complete Lattices}

Let $\mathbf{L}$ be a complete lattice. An interval triangular conorm ( $t$-conorm, for short) is a function $S: L^{2} \rightarrow L$ that is commutative, associative, monotonic and has 0 as the neutral element.

It follows from the work proposed in [5] that an interval t-conorm may be considered as an interval representation of a t-conorm. This interval generalization of a t-conorm on the unit interval lattice fits the fuzzy principle and the interval membership degree may be thought as an approximation of the exact degree.

Proposition 4.1. If $S$ is a t-conorm on the complete lattice $\mathbf{L}$ then $\widehat{S}: \mathbb{L}^{2} \rightarrow \mathbb{L}$ is a $t$-conorm on the complete lattice $\mathbb{I} \mathbf{L}$.

Proof. It is analogous to [6, Theorem 5.1].

Proposition 4.2. Let $S$ be a t-conorm on the complete lattice $\mathbf{L}$ and $\widehat{S}: \mathbb{L}^{2} \rightarrow$ $\mathbb{L}$ be the corresponded interval t-conorm on the interval lattice $\mathbb{I L}$. Based on the monotonicity of t-conorms, $\widehat{S}$ can be expressed by:

$$
\widehat{S}(X, Y)=[S(\underline{X}, \underline{Y}), S(\bar{X}, \bar{Y})] .
$$

Proof. Since, for each $x \in X$ and $y \in Y$, it holds that $\underline{X} \leq_{L} x \leq_{L} \bar{X}$ and $\underline{Y} \leq_{L} y \leq_{L} \bar{Y}$, then, based on the monotonicity of the t-conorm $S$, it follows that $S(\underline{X}, \underline{Y}) \leq_{L} S(x, y) \leq_{L} S(\bar{X}, \bar{Y})$. So, $S(\underline{X}, \underline{Y})$ is the minimal element and $S(\bar{X}, \bar{Y})$ is the maximal element (and then they are the infimum and supremum, repectively) of $\{S(x, y) \mid x \in X$ and $y \in Y\}$. Thus, by Eq. (3.1), it follows that $[S(\underline{X}, \underline{Y}), S(\bar{X}, \bar{Y})]=\widehat{S}(X, Y)$.

\section{Fuzzy Negations on Complete Lattices}

A function $N: L \rightarrow L$ is a fuzzy negation on the complete lattice $\mathbf{L}$ if

N1 : $N(0)=1$ and $N(1)=0$;

N2 : If $y \leq_{L} x$ then $N(x) \leq_{L} N(y)$; 
Fuzzy negations satisfying the involutive property are called strong fuzzy negations $[20,7]$ on $\mathbf{L}$, i.e.:

N3 : $N(N(x))=x, \forall x \in L$.

One can introduce a partial order on fuzzy negations in a natural way. For fuzzy negations $N_{1}$ and $N_{2}$, define $N_{1} \leq N_{2}$ if, for each $x \in L, N_{1}(x) \leq_{L} N_{2}(x)$.

A function $\mathbb{N}: \mathbb{L} \rightarrow \mathbb{L}$ is an interval fuzzy negation on the complete lattice $\mathbb{I} \mathbf{L}$ if the following conditions hold:

$\mathbb{N} 1: \mathbb{N}([0,0])=[1,1]$ and $\mathbb{N}([1,1])=[0,0]$

$\mathbb{N} 2 \mathbf{a}:$ If $Y \leq_{\mathbb{L}} X$ then $\mathbb{N}(X) \leq_{\mathbb{L}} \mathbb{N}(Y)$;

$\mathbb{N} 2 \mathbf{b}$ : If $X \subseteq Y$ then $\mathbb{N}(X) \subseteq \mathbb{N}(Y)$.

Interval fuzzy negations satisfying the involutive property $[7,20]$ on $\mathbb{L}$, are called strong interval fuzzy negations, that is:

$\mathbb{N} 3: \mathbb{N}(\mathbb{N}(X))=X, \forall X \in \mathbb{L}$.

Proposition 5.1. Let $N: L \longrightarrow L$ be a fuzzy negation on the complete lattice $\mathbf{L}$. $A$ characterization of $\widehat{N}: \mathbb{L} \rightarrow \mathbb{L}$ can be given by:

$$
\widehat{N}(X)=[N(\bar{X}), N(\underline{X})] .
$$

Proof. Since, for each $x \in X$, it holds that $\underline{X} \leq_{L} x \leq_{L} \bar{X}$, then, based on the antitonicity of $N$, it follows that $N(\bar{X}) \leq_{L} N(x) \leq_{L} N(\underline{X})$. So, $N(\bar{X})$ is the minimal element and $N(\underline{X})$ is the maximal element (and then they are the infimum and supremum, repectively) of $\{N(x) \mid x \in X\}$. Thus, by Eq. (3.1), it follows that $[N(\bar{X}), N(\underline{X})]=\widehat{N}(X)$.

In the next theorem, it is shown that the interval function $\widehat{N}$, presented in Prop. 5.1, satisfies the conditions of an interval fuzzy negation.

Theorem 5.1. Let $N: L \longrightarrow L$ be a fuzzy negation on the complete lattice $\mathbf{L}$. Then $\widehat{N}: \mathbb{L} \longrightarrow \mathbb{L}$ is a fuzzy negation on the complete lattice $\mathbb{I} \mathbf{L}$. Moreover, if $N$ is a strong fuzzy negation on $\mathbf{L}$ then $\widehat{N}$ is a strong fuzzy negation on $\mathbb{I} \mathbf{L}$.

Proof. It follows that:

$\mathbb{N} 1$ : It is trivial.

$\mathbb{N} 2 a$ : If $Y \leq_{\mathbb{L}} X$ then it holds that $\underline{Y} \leq_{L} \underline{X}$ and $\bar{Y} \leq_{L} \bar{X}$. So, by $\mathbf{N} 2$, it follows that $N(\underline{X}) \leq_{L} N(\underline{Y})$ and $N(\bar{X}) \leq_{L} N(\bar{Y})$. Therefore, one has that $[N(\bar{X}), N(\underline{X})] \leq_{\mathbb{L}}[N(\bar{Y}), N(\underline{Y})]$ and then, by Prop. 5.1, it follows that $\widehat{N}(X) \leq_{\mathbb{L}} \widehat{N}(Y)$.

$\mathbb{N} 2 b$ : If $X \subseteq Y$ then, it holds that $\underline{Y} \leq_{L} \underline{X}$ and $\bar{X} \leq_{L} \bar{Y}$. So, by $\mathbf{N} 2$, it follows that $N(\underline{X}) \leq_{L} N(\underline{Y})$ and $N(\bar{Y}) \leq_{L} N(\bar{X})$. Therefore, one has that $[N(\bar{X}), N(\underline{X})] \subseteq[N(\bar{Y}), N(\underline{Y})]$ and then, by Prop. 5.1, $\widehat{N}(X) \subseteq \widehat{N}(Y)$. 
$\mathbb{N} 3$ : Considering that $N$ is a strong fuzzy negation, by Prop. 5.1 and the involution of $N$, it holds that $\widehat{N} \circ \widehat{N}(X)=\widehat{N}([N(\bar{X}), N(\underline{X})])=[N(N(\underline{X})), N(N(\bar{X}))]=$ $X$.

\section{Fuzzy Implications}

Let $\mathbf{L}$ be a complete lattice. A binary function $I: L^{2} \longrightarrow L$ is a fuzzy implication on $\mathbf{L}$ if $I$ satisfies the minimal boundary conditions:

$$
I(1,1)=I(0,1)=I(0,0)=1 \text { and } I(1,0)=0 .
$$

Some reasonable properties may be required for fuzzy implications. The properties considered in this paper are listed below:

I1 : If $x \leq_{L} z$ then $I(x, y) \geq_{L} I(z, y)$;

I2 : If $y \leq_{L} z$ then $I(x, y) \leq_{L} I(x, z)$;

I3 : $I(1, x)=x$ (left neutrality principle);

I4 : $I(x, I(y, z))=I(y, I(x, z))$ (exchange principle);

I5 : $I(x, y)=I(N(y), N(x)$ ), with respect to a fuzzy negation $N$ (contraposition law).

Several definitions for fuzzy implication together with related properties have been given for the unit interval lattice $[7,13,30,31]$. In these definitions, a fuzzy implication should have the same behavior than the classical implication for the crisp case.

Proposition 6.1. Let $I: L^{2} \longrightarrow L$ be a fuzzy implication on a complete lattice $\mathbf{L}$ satisfying the properties $\mathbf{I} 1$ and $\mathbf{1 2}$. The interval fuzzy implication $\widehat{I}$ can be expressed as

$$
\widehat{I}(X, Y)=[I(\bar{X}, \underline{Y}), I(\underline{X}, \bar{Y})] .
$$

Proof. If $\underline{X} \leq_{L} x \leq_{L} \bar{X}$ and $\underline{Y} \leq_{L} y \leq_{L} \bar{Y}$ then, by $\mathbf{I 1}$ and I2, it follows that $I(\bar{X}, \underline{Y}) \leq_{L} I(x, y) \leq_{L} I(\underline{X}, \bar{Y})$, and then $\mathbb{I}=\{I(x, y): x \in X, y \in Y\} \subseteq \widehat{I}(X, Y)$. Since $I(\bar{X}, \underline{Y}), I(\underline{X}, \bar{Y}) \in \mathbb{I}$, then they are the infimum and the supremum of $\mathbb{I}$, respectively.

\subsection{S-implications and the Interval Constructor}

Let $S$ be a t-conorm and $N$ be a fuzzy negation on the complete lattice $\mathbf{L}$. Then the fuzzy implication called S-implication [20] on $\mathbf{L}$ is given by:

$$
I_{S, N}(x, y)=S(N(x), y) .
$$


An S-implication arises from the notion of disjunction and negation using the corresponding tautology of classical logic. More specifically, S-implications are based on the classical logical equivalence: $\alpha \rightarrow \beta \equiv \neg \alpha \vee \beta$.

One can notice that in some works (e.g., in $[1,7,13])$, an S-implication requires a strong fuzzy negation. In this case, the S-implication is called a strong S-implication. The main results relating S-implications and the properties $\mathbf{I 1}, \ldots, \mathbf{I} \mathbf{5}$ are considered below.

Proposition 6.2. Let $I: L^{2} \rightarrow L$ be a fuzzy implication. I is a strong $S$-implication on $\mathbf{L}$ if and only if $N_{I}(x)=I(x, 0)$ is a strong fuzzy negation and the properties $\mathbf{I} 1$ and $\mathbf{I} 4$ are satisfied.

Proof. It is analogous to [1, Theorem 2.6].

Theorem 6.1. Let $I: L^{2} \rightarrow L$ be a fuzzy implication. I is a strong S-implication on $\mathbf{L}$ if and only if the properties $\mathbf{I 1}$ or $\mathbf{I 2}, \mathbf{I 3}, \mathbf{I 4}$, and $\mathbf{I 5}$ (with respect to a strong fuzzy negation $N$ ) are satisfied.

Proof. It is analogous to [1, Theorem 2.8].

Considering any fuzzy implication on an arbitrary complete lattice $\mathbf{L}$, it is always possible to obtain an interval fuzzy implication canonically. An interval fuzzy implication satisfies the optimality property and preserves the same properties satisfied by a fuzzy implication. In the following, the best interval representation of a fuzzy implication is shown to be an inclusion-monotonic function in both arguments. The related proofs are straightforward, following from the definition of $\widehat{I}$ as a particular case of the Eq. (3.1).

Proposition 6.3. If I is a fuzzy implication on $\mathbf{L}$ then $\widehat{I}$ is a fuzzy implication on IIL.

Proposition 6.4. Let I be a fuzzy implication on $\mathbf{L}$. Then, for each $X_{1}, X_{2}, Y_{1}, Y_{2} \in$ $\mathbb{L}$, if $X_{2} \subseteq X_{1}$ and $Y_{1} \subseteq Y_{2}$ then it holds that $\widehat{I}\left(X_{1}, Y_{1}\right) \subseteq \widehat{I}\left(X_{2}, Y_{2}\right)$.

Proof. Suppose that $X_{2} \subseteq X_{1}$ and $Y_{1} \subseteq Y_{2}$. Thus, one has that: (i) $\underline{X}_{1} \leq_{L}$ $\underline{X}_{2} \leq_{L} \bar{X}_{2} \leq_{L} \bar{X}_{1}$ and, based on properties $\mathbf{I}_{1}$ and $\mathbf{I}_{2}$, it holds that $I\left(\underline{X}_{2}, \underline{Y}_{2}\right) \leq_{L}$ $I\left(\underline{X}_{1}, \underline{Y}_{2}\right) \leq I\left(\underline{X}_{1}, \underline{Y}_{1}\right)$; (ii) $\underline{Y}_{2} \leq_{L} \underline{Y}_{1} \leq_{L} \bar{Y}_{1} \leq_{L} \bar{Y}_{2}$ and, based on properties $\mathbf{I}_{1}$ and $\mathbf{I}_{2}$, it follows that $I\left(\bar{X}_{1}, \bar{Y}_{1}\right) \leq_{L} I\left(\bar{X}_{2}, \bar{Y}_{1}\right) \leq I\left(\bar{X}_{2}, \bar{Y}_{2}\right)$. Then, onde has that $\inf \left\{I(x, y) \mid x \in X_{2}, y \in Y_{2}\right\} \leq_{L}$ inf $\left\{I(x, y) \mid x \in X_{1}, y \in Y_{1}\right\}$ and $\sup \{I(x, y) \mid x \in$ $\left.X_{1}, y \in Y_{1}\right\} \leq_{L} \sup \left\{I(x, y) \mid x \in X_{2}, y \in Y_{2}\right\}$. It follows that $I\left(X_{1}, Y_{1}\right) \subset I\left(X_{2}, Y_{2}\right)$.

Theorem 6.2. Let I be a fuzzy implication on $\mathbf{L}$. If I satisfies a property $\mathrm{I} k$, for some $k=1, \ldots, 5$, then $\widehat{I}$ also satisfies the property $\mathbb{I} k$.

Proof. It follows that:

$\mathbb{I} 1$ : If $u \in \widehat{I}(Z, Y)$, there exist $z \in Z$ and $y \in Y$ such that $u \leq_{L} I(z, y)$. If $X \leq_{\mathbb{L}} Z$ then, by remark $1(i)$, there exists $x \in X$ such that $x \leq_{L} z$ and thus, by I1, it follows that $I(x, y) \geq_{L} I(z, y) \geq_{L} u$. On the other hand, if $v \in \widehat{I}(X, Y)$, 
there exist $x \in X$ and $y \in Y$ such that $v \geq_{L} I(x, y)$. If $X \leq_{\mathbb{L}} Z$ then, by remark $1(i)$, there exists $x \in X$ such that $x \leq_{L} z$ and therefore, by I1, $v \geq_{L} I(x, y) \geq_{L} I(z, y)$. So, one has that $\widehat{I}(X, Y) \geq_{\mathbb{L}} \widehat{I}(Z, Y)$.

I2 : If $u \in \widehat{I}(X, Y)$, there exist $x \in X$ and $y \in Y$ such that $u \leq_{L} I(x, y)$. If $Y \leq_{\mathbb{L}} Z$ then, by remark $1(i)$, there exists $y \in Y$ such that $y \leq_{L} z$ and therefore, by $\mathbf{I} 2, u \leq_{L} I(x, y) \leq_{L} I(x, z)$. Analogously, if $v \in \widehat{I}(X, Z)$, there exist $x \in X$ and $z \in Z$ such that $v \geq_{L} I(x, z)$. If $Y \leq_{\mathbb{L}} Z$ then, by remark $1(i)$, there exists $y \in Y$ such that $y \geq_{L} z$ and therefore, by $\mathbf{I 2}$, it follows that $I(x, y) \leq_{L} I(x, z) \leq_{L} v$. So, by remark $1(i)$, one has that $\widehat{I}(X, Y) \leq_{\mathbb{L}} \widehat{I}(X, Z)$.

$\mathbb{I} 3$ : Trivially, by $\mathbf{I} 3$, for each $x \in X$, it holds that $I(1, x)=x$, and then $\{I(1, x)$ : $x \in X\}=X$. Thus, since $\widehat{I}([1,1], X)$ is the narrowest interval containing $\{I(1, x): x \in X\}$, it follows that $\widehat{I}([1,1], X)=X$.

II : If $u \in \widehat{I}(X, \widehat{I}(Y, Z))$ then there exist $x \in X, y \in Y$ and $z \in Z$ such that $u \leq_{L} I(x, I(y, z))$. But, by $\mathbf{I} 4$, it holds that $I(x, I(y, z))=I(y, I(x, z)) \in$ $\widehat{I}(Y, \widehat{I}(X, Z))$. On the other hand, if $v \in \widehat{I}(Y, \widehat{I}(X, Z))$ then there exist $x \in X$, $y \in Y$ and $z \in Z$ such that $v \leq_{L} I(y, I(x, z))$. But, by I4, it holds that $I(y, I(x, z))=I(x, I(y, z)) \in \widehat{I}(X, \widehat{I}(Y, Z))$. So, by remark $1(i i)$, one has that $\widehat{I}(X, \widehat{I}(Y, Z))=\widehat{I}(Y, \widehat{I}(X, Z))$.

I5 : If $u \in \widehat{I}(X, Y)$ then $u \leq_{L} I(x, y)$, for some $x \in X$ and $y \in Y$. But, by property I5, it holds that $I(x, y)=I(N(y), N(x))$. On the other hand, If $v \in \widehat{I}(\widehat{N}(X), \widehat{N}(Y))$ then $v \leq_{L} I(N(x), N(y))$, for some $x \in X$ and $y \in Y$. But, by property I5, $I(N(y), N(x))=I(x, y)$. So, by remark $1(i i)$, it follows that $\widehat{I}(X, Y)=\widehat{I}(\widehat{N}(X), \widehat{N}(Y))$.

\subsection{S-implications on Complete Lattices and the Interval Constructor}

Theorem 6.3. Let $S$ be a t-conorm on $\mathbf{L}$ and $N$ be a fuzzy negation on $\mathbf{L}$. Then it holds that $I_{\widehat{S}, \widehat{N}}=\widehat{I_{S, N}}$.

Proof. Consider $X, Y \in \mathbb{L}$. Applying Eq. (6.2), one has that $I_{\widehat{S}, \widehat{N}}(X, Y)=$ $\widehat{S}(\widehat{N}(X), Y)$. By Eq. (5.1), it follows that $I_{\widehat{S}, \widehat{N}}(X, Y)=\widehat{S}([N(\bar{X}), N(\underline{X})], Y)$. Based on Eq. (4.1), it holds that $\widehat{S}([N(\bar{X}), N(\underline{X})], Y)=[S(N(\bar{X}), \underline{Y}), S(N(\underline{X}), \bar{Y})]$, and due to the definition of an S-implication in Eq. (6.2) we obtain $[S(N(\bar{X}), \underline{Y})$, $S(N(\underline{X}), \bar{Y})]=\left[I_{S, N}(\bar{X}, \underline{Y}), I_{S, N}(\underline{X}, \bar{Y})\right]$, which, by Eq. (6.1), is equal to $\widehat{I_{S, N}}(X, Y)$.

Corollary 6.3. If $I$ is an S-implication on $\mathbf{L}$ then $\widehat{I}$ is an $S$-implication on $\mathbb{I} \mathbf{L}$.

Proof. If $I$ is an S-implication on $\mathbf{L}$, then there exist a t-conorm $S$ and a fuzzy negation $N$ on $\mathbf{L}$ such that $I=I_{S, N}$. By Theorem 6.3 , it follows that $\widehat{I}=I_{\widehat{S}, \widehat{N}}$, which is an S-implication on $\mathbb{I} \mathbf{L}$. 
The above results together with Theorem 6.3 state the commutativity of the diagram in Fig. 1, where $\mathcal{C}(S)_{\mathbf{L}}$ denotes the class of t-conorms on $\mathbf{L}, \mathcal{C}(N)_{\mathbf{L}}$ indicates the class of fuzzy negations on $\mathbf{L}$ and $\mathcal{C}(I)_{\mathbf{L}}$ is the class of S-implications on $\mathbf{L}$.

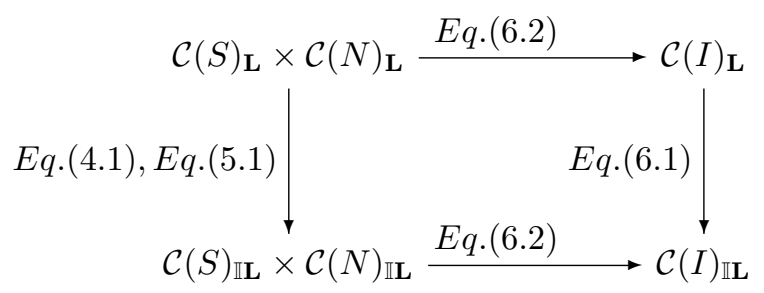

Figure 1: Commutative diagram relating S-implications on $\mathbf{L}$ with S-implications on $\mathbb{I} \mathbf{L}$

\section{Conclusion}

The use of t-norms and t-conorms can be considered vital for more flexible and reliable fuzzy logic controllers. From t-norms and t-conorms, it is possible to derive several fuzzy implication functions (e.g, S-implications, R-implications, QLimplications, D-implications), which are important not only because they are used in representing "If ... then" rules in fuzzy systems, but also because their use in performing inferences in approximate reasoning and fuzzy control.

This paper complements the results of previous works $[4,5,6]$ related to the approach of interval fuzzy logic, which considers interval mathematics to deal with the uncertainty of membership degrees of fuzzy sets. We presented an approach of interval fuzzy logic based on complete lattices. In particular, we introduced a generalization of t-conorms, fuzzy negations and S-implication for arbitrary complete lattices. Some general properties of S-implications on complete lattices were analyzed. We also showed that the interval extensions of t-conorms, fuzzy negations and S-implications on complete lattices preserve the optimality property, being the best interval representations of these fuzzy connectives.

The results presented in this paper are important not only to analyze deductive systems in mathematical depth but also to provide foundations of methods based on interval fuzzy logic. They integrate two important features: the accuracy criteria and the optimality property of interval computations, and a formal mathematical theory for the representation of uncertainty, concerned with the fuzzy set theory. The former gives a more reliable modelling of real systems and the latter is crucial for their management and control.

\section{Acknowledgments}

This work was partially supported by CNPq (470871/04-0 and 473201/07-0). We are grateful to the referees for their valuable comments that helped us to improve the paper. 
Resumo. O objetivo deste trabalho é apresentar uma abordagem da lógica fuzzy intervalar baseada en reticulados completos. Em particular, extensões de t-normas, negações fuzzy e S-implicações do intervalo unitário para um reticulado completo arbitrário são apresentadas. Algumas propriedades gerais de S-implicações sobre reticulados completos são analisadas. Mostra-se que as extensões intervalares de t-norms, negações fuzzy e S-implicações sobre reticulados completos preservam a propriedade de otimalidade e conduzem à melhor representação intervalar desses conectivos fuzzy.

\section{References}

[1] M. Baczyński, B. Jayaram, On the characterization of (S,N)-implications generated from continuous negations, Fuzzy Sets and Systems, 158 (2007), 17131727.

[2] B. De Baets, R. Mesiar, Triangular norms on product lattices, Fuzzy Sets and Systems, 104 (1999), 61-75.

[3] L.V. Barboza, G.P. Dimuro, R.H.S. Reiser, Towards interval analysis of the load uncertainty in power electric systems, in "Proc. of the IEEE 8th Intl. Conf. on Probability Methods Applied to Power Systems", pp. 1-6, IEEE, Ames, 2004.

[4] B.C. Bedregal, H.S. Santos, R. Callejas-Bedregal, T-norms on bounded lattices: t-norm morphisms and operators, in "Proc. of the IEEE Intl. Conf. on Fuzzy Systems", pp. 22-28, IEEE, Vancouver, 2006.

[5] B.C. Bedregal, A. Takahashi, The best interval representation of t-norms and automorphisms, Fuzzy Sets and Systems, 157, No. 24 (2006), 3220-3230.

[6] B.C. Bedregal, A. Takahashi, Interval valued versions of t-conorms, fuzzy negations and fuzzy implications, in "Proc. of the IEEE Intl. Conf. on Fuzzy Systems", pp. 553-559, IEEE, Vancouver.

[7] H. Bustince, P. Burilo, F. Soria, Automorphism, negations and implication operators, Fuzzy Sets and Systems, 134 (2003), 209-229.

[8] G. De Cooman, E. Kerre, Order norms on bounded partially ordered sets, Journal Fuzzy Mathematics, 2 (1994), 281-310.

[9] C. Cornelis, G. Deschrijver, E.E. Kerre, Advances and challenges in intervalvalued fuzzy logic, Fuzzy Sets and Systems, 157 (2006), 622-627.

[10] G. Deschrijver, A representation of t-norms in interval-valued L-fuzzy set theory, Fuzzy Sets and Systems, 159, No. 13 (2008), 1597-1618.

[11] G.P. Dimuro, A.C.R. Costa, Interval-based Markov Decision Processes for regulating interactions between two agents in multi-agent systems, in "Applied Parallel Computing" (J. Dongarra, K. Madsen and J. Wasniewski, eds.), LNCS, No. 3732, pp. 102-111, Springer, Berlin, 2006. 
[12] D. Dubois and H. Prade, Random sets and fuzzy interval analysis, Fuzzy Sets and Systems, 12 (1991), 87-101.

[13] J.C. Fodor, On fuzzy implication operators, Fuzzy Sets and Systems, 42 (1991), 293-300.

[14] M. Gehrke, C. Walker, E. Walker, Some comments on interval valued fuzzy sets, International Journal of Intelligent Systems, 11 (1996), 751-759.

[15] J. Goguen, L-fuzzy sets, Mathematics Analisys and Applications, 18 (1967), 145-174.

[16] P. Hájek, Basic fuzzy logic and BL-algebras, Soft Computing, 2 (1998), 124128.

[17] E. Hansen, Sharpness in interval computations, Reliable Computing, 3, (1997), 1-29.

[18] T. Hickey, Q. Ju, M. Emdem, Interval arithmetic: from principles to implementation, Journal of the ACM, 48, No. 5 (2001), 1038-1068.

[19] R.B. Keafort, V. Kreinovich (eds.), "Applications of Interval Computations", Kluwer, Boston, 1996.

[20] E.P. Klement, R. Mesiar, E. Pap, "Triangular Norms", Kluwer, Dordrecht, 2000.

[21] U. Kulisch, W. Miranker, "Computer Arithmetic in Theory and Practice", Academic Press, 1981.

[22] W.A. Lodwick, Preface, Reliable Computing, 10, No. 4 (2004), 247-248.

[23] S. Mitra, S. K. Pal, Fuzzy Sets in Pattern Recognition and Machine Intelligence, Fuzzy Sets and Systems, 156 (2005), 381-386.

[24] R.E. Moore, W. Lodwick, Interval analysis and fuzzy set theory, Fuzzy Sets and Systems, 135, No. 1 (2003), 5-9.

[25] R.E. Moore, "Methods and Applications of Interval Analysis", SIAM, Philadelphia, 1979.

[26] S. Ray, Representation of a boolean algebra by its triangular norms, Matheware \& Soft Computing, 4 (1997), 63-68.

[27] R.H.N. Santiago, B.C. Bedregal, B.M. Acióly, Interval Representations, TEMA - Tendências em Matemática Aplicada e Computacional, 5, No. 2 (2004), 317326.

[28] R.H.N. Santiago, B.C. Bedregal, B.M. Acióly, Formal aspects of correctness and optimality of interval computations, Formal Aspects of Computing, 18, No. 2 (2006), 231-243. 
[29] Z.D. Wang, Y.D. Yu, Pseudo t-norms and implication operators on a complete brouwerian lattice, Fuzzy Sets and Systems, 132 (2002), 113-124.

[30] R.R. Yager, On the implication operator in fuzzy logic, Information Sciences, 31 (1983), 141-164.

[31] R.R. Yager, On some new classes of implication operators and their role in approximate reasoning, Information Sciences, 167 (2004), 193-216.

[32] L.A. Zadeh, Fuzzy probabilities, Information Processing and Management, 20 (1984), 363-372. 